\title{
Dyspepsia management in the millennium: to test and treat or not?
}

\section{B C Delaney}

Test and treat followed by endoscopy for non-responders was less cost effective than empiric proton pump inhibitor (PPI) treatment followed by endoscopy or with strategies of test and treat followed by empiric PPI (or vice versa).

$\mathrm{D}$ ecision analysis can be used to bridge the gap between evidence and practice. It may be used to highlight the best choice between competing management strategies where no direct trial evidence is available or add additional information, for example cost data, to extrapolate from available trial evidence. ${ }^{1}$ The use of sensitivity analysis, changing the parameters such as the prevalence of Helicobacter pylori, is particularly helpful in establishing an "envelope" within which a particular strategy is cost effective. Trials, metaanalyses, and modelling can be seen as working together to create a practical quantified evidence base.

Cochrane reviews on the management of dyspepsia, ${ }^{2}$ therapies for non-ulcer dyspepsia, ${ }^{34}$ and the treatment of endoscopy negative and uninvestigated gastrooesophageal reflux disease ${ }^{5}$ have created a sufficiently rich evidence base upon which to build sophisticated models incorporating alternative approaches to both investigative and empirical treatment strategies in dyspepsia. Health service researchers in both the $\mathrm{USA}^{6}$ and the $\mathrm{UK}^{7}$ have now published models that in spite of methodological differences (discrete event simulation $v$ Monte Carlo simulation $)^{8}$ come to similar conclusions as to the cost effectiveness of the H pylori "test and treat strategy" compared with endoscopy and empiric proton pump inhibitor (PPI) treatment in young patients without alarm symptoms.

As dyspepsia is a chronic relapsing disorder, the management of patients with persisting symptoms is as important as the initial management strategy. This is difficult to capture in randomised trials where complex algorithms and planned follow up may take trials too far from everyday practice. The effect of possible "follow on" strategies can be explored by modelling. Both the UK and US models allowed for endoscopy of treatment failures. The UK model did not use the same base case as the US model, "test and treat" followed by endoscopy for treatment failures, as it was assumed that in UK primary care a trial of PPI would always be interposed before endoscopy referral. The US analysis is useful in showing that this early use of

Spiegel BMR, Vakil NB, Ofman JJ. Dyspepsia management in primary care-a decision analysis of competing strategies. Gastroenterology 2002; 122: 1270-85.

Background: Several consensus statements have recommended Helicobacter pylori testing and eradication (test and treat) followed by endoscopy for non-responders for the management of simple uninvestigated dyspepsia, particularly in patients aged $<45$ years. However, recent trials have suggested that this may not be such a good strategy.

Question: For uninvestigated dyspepsia, how cost effective is test and treat with endoscopy for non-responders compared with empiric proton pump inhibitor (PPI) treatment followed by endoscopy or with strategies of test and treat followed by empiric PPI (or vice versa), with endoscopy reserved for persistently symptomatic patients?

Design and methods: Decision analysis evaluating the cost effectiveness of the four treatment strategies in a hypothetical cohort of patients younger than 45 years presenting in primary care for the first time with dyspepsia. Patients with alarm symptoms and those taking non-steroidal anti-inflammatory drugs were excluded, as were patients with predominant symptoms of gastro-oesophageal reflux. The model used serology for $\mathrm{H}$ pylori testing, 14 days of $\mathrm{H}$ pylori eradication (cost \$304), six weeks of treatment with a PPI, and endoscopy for persistently symptomatic patients.

Results: Test and treat with endoscopy for non-responders was the most costly strategy and resulted in only $75 \%$ of patients being symptom free at one year. Test and treat followed by PPI and PPI followed by test and treat were equally effective (84\% symptom free at one year) but performing test and treat first was cheaper and therefore slightly more cost effective. PPI followed by endoscopy for non-responders was the cheapest strategy but resulted in only $78 \%$ of patients being symptom free at one year. These results were sensitive to the costs of the PPI and to the prevalence of $\mathrm{H}$ pylori, with the PPI followed by endoscopy strategy becoming the more cost effective when $\mathrm{H}$ pylori prevalence fell to $12 \%$.

Conclusions: Even when the baseline assumptions were varied, test and treat followed by endoscopy for non-responders was less cost effective than the three strategies incorporating a period of PPI treatment. Test and treat followed by PPI with endoscopy for non-responders cost $\$ 2500$ less per additional symptomatic cure than the current guidelines recommending test and treat with endoscopy for non-responders.

endoscopy is indeed less cost effective than interposing a trial of PPI therapy.

The message from both models is that endoscopy adds little to the management of dyspeptic patients under the age of 55 years without alarm symptoms for malignancy, and that the most important uncertainty is whether to test and treat for $H$ pylori initially, or after PPI therapy, or not at all. The balance depends on the prevalence of $H$ pylori related and acid related disease in the population and the relative costs of endoscopy, eradication therapy, and PPIs.

Recent trials confirm that "test and treat" is as effective and less costly than endoscopy based management in patients referred to a dyspepsia clinic. ${ }^{9}$ A recent Canadian trial has also shown that "test and treat" in primary care is more effective than initial PPI therapy at preventing recurrence of dyspeptic symptoms at one year in $H$ pylori positive patients $(\mathrm{NNT}=7) .{ }^{10}$ A significant difference in costs was not shown but exclusion of $H$ pylori negative patients makes the economic comparison incomplete.

In the UK, the lower cost of $H$ pylori eradication therapy relative to endoscopy and PPIs should lower the threshold for a "test and treat" approach. A large primary care based MRC funded trial 
(CUBE) which has just started aims to determine the cost effectiveness of initial "test and treat" compared with initial empirical acid suppression. Until there is more evidence, either approach is justifiable.

Gut 2003;52:10-11

Author's affiliation

B C Delaney, Primary Care Clinical Sciences

Building, University of Birmingham, Edgbaston, Birmingham B15 2T, UK;

b.c.delaney@bham.ac.uk

\section{REFERENCES}

1 Lilford RJ, Pauker SG, Braunholtz DA, et al Decision analysis and the implementation of research findings. BM 1998;317:405-9.
2 Delaney BC, Innes MA, Deeks J, et al. Initial management strategies for dyspepsia (full Cochrane Review). In: The Cochrane Library issue 3. Oxford: Update Software, 2001.

3 Soo S, Moayyedi P, Deeks J, et al.

Pharmacological interventions for non-ulcer dyspepsia (full Cochrane Review). In: The Cochrane Library, issue 2. Oxford: Update Software, 2000.

4 Soo S, Moayyedi P, Deeks J, et al. Helicobacter pylori eradication for non-ulcer dyspepsia (full Cochrane Review). In: The Cochrane Library, issue 2. Oxford: Update Software, 2000.

5 van Pinxteren B, Numans ME, Bonis PA, et al. Short-term treatment with proton pump inhibitors, $\mathrm{H} 2$-receptor antagonists and prokinetics for gastro-oesophageal reflux disease-like symptoms and endoscopy negative reflux disease. Cochrane Database of Systematic Reviews 2000; CD002095.

6 Spiegel BM, Vakil NB, Ofman JJ. Dyspepsia management in primary care: a decision analysis of competing strategies.

Gastroenterology 2002;122:1270-85.

7 Delaney BC, Moayyedi P, Deeks J, et al. The management of dyspepsia: A systematic review. Health Technol Assess 2000;4: 1-187.

8 Sonnenberg FA, Beck JR. Markov models in medical decision making: a practical guide. Med Decis Making 1993;13:322-38.

9 McColl KEL, Murray LS, Gillen D, et al.

Randomised trial of endoscopy with testing for Helicobacter pylori compared with

non-invasive $\mathrm{H}$ pylori testing alone in the

management of dyspepsia. BM 2002;324:999.

10 Chiba N, Veldhuyzen van Zanten SJO, Sinclair $\mathrm{P}$, et al. Treating Helicobacter pylori infection in primary care patients with uninvestigated dyspepsia: the Canadian adult dyspepsia empiric treatment Helicobacter pylori positive (CADET-Hp) randomised controlled trial. BM 2002;324:1012. 